\title{
Coherent State Representations of Nilpotent Lie Groups
}

\author{
Henri Moscovici \\ Institute for Atomic Physics, Bucharest, Romania
}

\begin{abstract}
The coherent state representations of a connected and simply connected nilpotent Lie group are characterized in terms of the Kirillov correspondence, as being those irreducible unitary representations whose associated orbits under the coadjoint representation are linear varieties.
\end{abstract}

\section{Introduction}

The concept of coherent states, originally related to the Weyl representation of the Heisenberg group, has been extended by Perelomov [8] to a general group theoretical setting, involving an irreducible unitary representation of an arbitrary Lie group. We shall adopt here a slightly modified version of Perelomov's definition, which reveals from the very beginning the classical phase space that parametrizes the coherent states. Our concern in this paper is to determine which irreducible unitary representations of an arbitrary nilpotent Lie group admit coherent states. The interest for nilpotent groups is motivated not only by the fact that the Heisenberg group belongs to this class, but also by the existence of the Kirillov orbital description for the unitary dual of such a group. Indeed, we find that, in terms of the Kirillov correspondence, the irreducible unitary representations of a connected and simply connected nilpotent Lie group which admit coherent states are associated with those orbits of its coadjoint representation which are of the simplest geometric form, namely linear varities. In other words, they are essentially square integrable representations, like in the special case of Heisenberg groups. Moreover, we show that, in a certain sense, the only classical phase space which may support a system of coherent states for such a representation is precisely the corresponding orbit.

1.

Let $G$ denote a locally compact group and $X$ a $G$-homogeneous space which admits an invariant measure $\mu_{X}$. After fixing a point $0 \in X$, we shall identify $X$ to the quotient space $G / G_{0}$, where $G_{0}$ is the isotropy subgroup of $G$ at 0 , and the measure 
$\mu_{X}$ to the quotient measure $\mu_{G} / \mu_{0}$, where $\mu_{G}$ and $\mu_{0}$ are left Haar measures on $G$ and $G_{0}$, respectively.

1.1. Definition. Let $\pi$ be an irreducible unitary representation of $G$ on a Hilbert space $\mathscr{H}$. A family $\left\{E_{p} ; p \in X\right\}$ of 1 -dimensional projections in $\mathscr{H}$ will be called a $\pi$-system of coherent states based on $X$, if the following conditions are satisfied:

$\left(\mathrm{CS}_{1}\right) E_{g \cdot p}=\pi(g) \cdot E_{p} \cdot \pi(g)^{-1}$ for any $g \in G$ and $p \in X$; $\left(\mathrm{CS}_{2}\right)$ there exists a nonzero vector $\psi \in \mathscr{H}$, such that

$$
\int\left\langle E_{p} \psi, \psi\right\rangle d \mu_{X}(p)<\infty \text {. }
$$

Let us add some comments on this definition. In view of $\left(\mathrm{CS}_{1}\right), E_{0} \cdot \pi(\gamma)=\pi(\gamma)$ - $E_{0}$ for any $\gamma \in G_{0}$. Therefore, if we choose a vector $\varphi_{0}$ in the range of $E_{0}$ with $\left\|\varphi_{0}\right\|$ $=1$, then $\varphi_{0}$ is semi-invariant with respect to $\pi \mid G_{0}$. More exactly, it has the property:

$\left(\mathrm{CS}_{1}^{\prime}\right) \pi(\gamma) \varphi_{0}=\lambda_{0}(\gamma) \varphi_{0}$ for all $\gamma \in G_{0}$, where $\lambda_{0}$ is a unitary character of $G_{0}$.

In addition, $E_{p} \psi=\left\langle\psi, \pi(g) \varphi_{0}\right\rangle \pi(g) \varphi_{0}$ for any $\psi \in \mathscr{H}$, when $g \in G$ is such that $p$ $=g \cdot 0$. In these terms, the property $\left(\mathrm{CS}_{2}\right)$ says that:

$\left(\mathrm{CS}_{2}^{\prime}\right)$ there exists a nonzero vector $\psi \in \mathscr{H}$, such that

$$
\int\left|\left\langle\psi, \pi(g) \varphi_{0}\right\rangle\right|^{2} d \mu_{X}(g \cdot 0)<\infty \text {. }
$$

Conversely, assume that $\varphi_{0} \in \mathscr{H}$ is a vector of norm one which satisfies the properties $\left(\mathrm{CS}_{1}^{\prime}\right)$ and $\left(\mathrm{CS}_{2}^{\prime}\right)$. Define $E_{p}$ to be the 1-dimensional projection associated with $\pi(g) \varphi_{0}$ for $p=g \cdot 0$. In view of $\left(\mathrm{CS}_{1}^{\prime}\right)$ the choice of $g$ in the fiber over $p$ is immaterial, so that $E_{p}$ depends indeed only on the point $p \in X$. Moreover, it is not difficult to check that the family $\left\{E_{p} ; p \in X\right\}$ thus defined forms a $\pi$-system of coherent states based on $X$ and this system is uniquely determined by the property that $\varphi_{0}$ is in the range of $E_{0}$.

It is now easily seen that our definition of coherent states differs from Perelomov's definition in only one point. Namely, we do not require to the isotropy subgroup $G_{0}$ to be maximal with the property that $\pi \mid G_{0}$ stabilizes the state defined by $\varphi_{0}$.

We shall not take the space here to disscuss the elementary properties of the coherent states introduced above, since they are essentially the expected ones. We will only retain, for later use, the fact expressed in the proposition which follows.

Let $\left\{E_{p} ; p \in X\right\}$ be a $\pi$-system of coherent states, $\varphi_{0}$ a vector of norm one in the range of $E_{0}$ and $\lambda_{0}$ the corresponding unitary character of the isotropy subgroup $G_{0}$. We shall denote by ind $\left(G_{0}, G, \lambda_{0}\right)$, or more simply $U^{0}$, the unitary representation of $G$ induced by $\lambda_{0}$. Recall that the Hilbert space of $U^{0}$, which will be denoted here $\mathscr{L}^{2}\left(G ; G_{0}, \lambda_{0}\right)$, consists of those complex functions on $G$ satisfying the relation $F(g \gamma)=\lambda_{0}(\gamma)^{-1} F(g)$ for $g \in G$ and $\gamma \in G_{0}$, which are square integrable on $X=G / G_{0}$, and that $G$ operates on $\mathscr{L}^{2}\left(G ; G_{0}, \lambda_{0}\right)$ by the formula $U^{0}(g) F(h)=F\left(g^{-1} h\right), g, h \in G$.

1.2. Proposition. Let $\pi$ be an irreducible unitary representation of $G$ which admits a system $\left\{E_{p} ; p \in X\right\}$ of coherent states based on $X=G / G_{0}$ and let $\lambda_{0}$ be the corresponding unitary character of $G_{0}$. Then $\pi$ is contained in the representation ind $\left(G_{0}, G, \lambda_{0}\right)$. 
Proof. For $\psi \in \mathscr{H}$, define $F_{\psi}$ to be the function on $G$ given by $F_{\psi}(g)=\left\langle\psi, \pi(g) \varphi_{0}\right\rangle$. Clearly, $F_{\psi}(g \gamma)=\lambda_{0}(\gamma)^{-1} F_{\psi}(g)$ for $g \in G, \gamma \in G_{0}$. Now we define an operator $D$ from $\mathscr{H}$ to $\mathscr{L}^{2}\left(G ; G_{0}, \lambda_{0}\right)$ as follows. The domain of $D$ consists of all $\psi \in \mathscr{H}$ such that $F_{\psi} \in \mathscr{L}^{2}\left(G ; G_{0}, \lambda_{0}\right)$, that is $\int\left|\left\langle\psi, \pi(g) \varphi_{0}\right\rangle\right|^{2} d \mu_{X}(g \cdot 0)<\infty$ and $D \psi=F_{\psi}$. It is not difficult to see that $D$ is a closed operator which intertwines $\pi$ and $U^{0}$. Moreover, since its domain is nonzero, $D$ is densely defined. By Schur's lemma, it follows that $D$ is a multiple of an isometry. In particular the domain of $D$ is the whole $\mathscr{H}$ and $\pi$ is unitarily equivalent to a subrepresentation of $U^{0}$. This proves the proposition.

Remark. Actually, the above arguments imply a little more than we said. Namely, $D$ being a multiple of an isometry, there exists a positive number $d$ such that $\left\langle D \psi_{1}\right.$, $\left.D \psi_{2}\right\rangle=d\left\langle\psi_{1}, \psi_{2}\right\rangle$ for all $\psi_{1}, \psi_{2} \in \mathscr{H}$. This means that $\int E_{p} d \mu_{X}(p)=d \cdot I$, where $I$ stands for the identity operator of $\mathscr{H}$.

2.

From now on, unless otherwise stated, $G$ will be a connected, simply connected nilpotent Lie group. Let $\mathfrak{g}$ denote its Lie algebra and $\mathfrak{g}$ * the dual vector space of $\mathfrak{g}$. $G$ acts on $\mathrm{g}^{*}$ by the coadjoint representation: $\operatorname{Ad}^{*}(g) f=f \cdot \operatorname{Ad}(g)^{-1}, g \in G, f \in \mathfrak{g}^{*}$. According to the Kirillov theory [3], all the irreducible unitary representations of $G$ can be constructed by the orbit method. More exactly, if we denote by $\pi_{O}$ the representation of $G$ associated with the orbit $O \in \mathfrak{g}^{*} / \operatorname{Ad}^{*}(G)$, then the assignment $O \mapsto \pi_{O}$ establishes a natural one-to-one correspondence between the space of orbits $\mathrm{g}^{*} / \operatorname{Ad}^{*}(G)$ and the unitary dual $\hat{G}$ of $G$. Finally, recall that any orbit $O$ is a symplectic manifold with respect to the Kirillov form $\omega_{O}$ and thus it possesses a canonical $G$-invariant measure $\mu_{o}$ given by the volume element $\omega_{o}^{n}$, where $n=1 / 2$ $\operatorname{dim} O$. Furthermore, $O$ is an algebraic variety in $\mathrm{g}^{*}$ which contains a linear variety of dimension at least $n$.

2.1. Proposition. Suppose that the orbit $O \in \mathfrak{g}^{*} / \mathrm{Ad}^{*}(G)$ is itself a linear variety. Then $\pi_{o}$ admits a system of coherent states based on $O$.

Proof. It has been noticed by Brezin ([1], Theorem 1.1) that $O$ is a linear variety if and only if $\pi_{o}$ defines a square integrable representation of the group $G / K$, where $K$ stands for the neutral component of the kernel of $\pi_{o}$. We may assume therefore that $\pi_{O}$ is square integrable. But then it follows from [6] that : i) $O=G / Z, Z$ being the center of $G$; ii) $\pi_{o} \mid Z$ is a multiple of a character $\lambda_{o} \in \hat{Z}$; iii) $\int\left|\left\langle\psi, \pi_{o}(g) \varphi\right\rangle\right|^{2} d \mu_{o}(g Z)$ $<\infty$, for any $\psi, \varphi$ in the Hilbert space $\mathscr{H}_{o}$ in which $\pi_{o}$ operates. It is now clear that the conditions $\left(\mathrm{CS}_{1}^{\prime}\right)$ and $\left(\mathrm{CS}_{2}^{\prime}\right)$ are satisfied. More precisely, any vector $\varphi \in \mathscr{H}_{O}$ with $\|\varphi\|=1$ defines a $\pi_{O^{-}}$-system of coherent states based on the orbit $O$.

3.

The point is that the converse assertion is also true.

3.1. Theorem. Let $\pi$ be an irreducible unitary representation of the connected, simply connected nilpotent Lie group $G$, and let $O_{\pi}$ be the corresponding orbit in $\mathrm{g}^{*} / \operatorname{Ad}^{*}(G)$. Suppose that $\pi$ admits a system of coherent states based on a connected, simply connected $G$-homogeneous space $X$. Then $O_{\pi}$ is a linear variety in $\mathrm{g}^{*}$, and $X$ and $O_{\pi}$ are isomorphic G-spaces. 
In view of Proposition 1.2, this theorem will follow from Lemma 3.5 which we shall prove below, after some preliminary remarks.

3.2. If $H$ and $K$ are closed, connected subgroups of $G$, then they are regularly related in the sense of Mackey [5].

This follows, for instance, first noting that $V=G / K$ is a real algebraic manifold on which the real algebraic group $H$ acts algebraically, and then using a result of Chevalley (quoted in [2], p. 183) which asserts that, under these assumptions, the quotient space $H \backslash V=H \backslash G / K$ is countably separated.

This remark will allow us to use the Mackey Subgroup Theorem ([5], Theorem 12.1), whenever we shall deal with a pair of closed, connected subgroups $H$ and $K$ of $G$. For convenience, let us recall that the Subgroup Theorem asserts that, in such a case, if $\varrho$ is a unitary representation of $H$, then

$$
\operatorname{ind}(H, G, \varrho) \mid K=\int_{H \backslash G / K}^{\oplus} \operatorname{ind}\left(K \cap H_{g}, K, \varrho_{g} \mid K \cap H_{g}\right) d v(\dot{g}),
$$

where $H_{g}=g^{-1} H g, \varrho_{g}(\gamma)=\varrho\left(g \gamma g^{-1}\right)$ for $\gamma \in H_{g}$, and $v$ is an "admissible" measure on $H \backslash G / K$. It should be noted that the unitary equivalence class of the representation $\operatorname{ind}\left(K \cap H_{g}, K, \varrho_{g} \mid K \cap H_{g}\right)$ depends only on the $H: K$ double coset $\dot{g}$ to which $g \in G$ belongs. Finally, the meaning of the term "admissible" measure attributed to $v$ is the following: pick a finite measure $\mu$ on $G$ equivalent to the Haar measure $\mu_{G}$, equip $H \backslash G / K$ with the quotient Borel structure and then set $v(E)=\mu\{g \in G ; \dot{g} \in E\}$ for any Borel subset $E$ of $H \backslash G / K$.

3.3. Let $\Gamma$ be a separable locally compact group of Type I and let $\varrho=\int_{B}^{\oplus} \varrho_{t} d \beta(t)$ be a direct integral decomposition of the unitary representation $\varrho$ of $\Gamma$ over the Borel measure space $(B, \beta)$. Assume that $\varrho$ contains an irreducible unitary representation $\pi$ of $\Gamma$. Then, there exists a measurable subset $E$ of $B$ with $\beta(E)>0$ such that $\pi$ is a subrepresentation of $\varrho_{t}$ for all $t \in E$.

This follows by routine arguments from direct integral theory. However, for the sake of completeness, we shall sketch a proof. Let $R(\varrho)$ be the von Neumann algebra generated by $\varrho(\Gamma), C(\varrho)$ its commutant and $Z(\varrho)=R(\varrho) \cap C(\varrho)$ the center of $R(\varrho)$. Further, let $P \in C(\varrho)$ be the projection associated with $\pi, \tilde{P} \in Z(\varrho)$ its central support and $\tilde{\pi}$ the corresponding subrepresentation of $\varrho$. Then, there exists a measurable subset $\tilde{E}$ of $B$ with $\beta(\tilde{E})>0$, such that $\tilde{\pi}=\int_{E}^{\oplus} \tilde{\pi}_{t} d \beta(t)$, where $\tilde{\pi}_{t}$ is contained in $\varrho_{t}$ for all $t \in E$. Since $\tilde{\pi}$ is quasi-equivalent to $\pi$, which is irreducible, $\tilde{\pi}$ is a multiple of $\pi$. It follows that almost all $\tilde{\pi}_{t}$ are multiples of $\pi$. Substracting if need a negligeable set from $\tilde{E}$, we get the desired subset $E$ of $B$.

3.4. Let $H$ be a closed, connected subgroup of the connected, simply connected, nilpotent Lie group $G$ and let $\chi$ be a unitary character of $H$. Assume that ind $(H, G, \lambda)$ contains a unitary character $\lambda$ of $G$. Then $H=G$ and $\chi=\lambda$.

Indeed, if $H \neq G$, by Theorem 8.2 in [5], $G / H$ would admit a finite $G$-invariant measure, which would imply compactness for $G / H$ [cf. Mostow, G. D., Homogeneous spaces with finite invariant measure. Ann. Math. 75, 17-37 (1962); Theorem 7.1]. But $G / H$ is easily seen to be diffeomorphic to $\mathbb{R}^{m}$, with $m=\operatorname{dim} G$ $-\operatorname{dim} H$. 
3.5. Lemma. Let $H$ be a closed, connected subgroup of the connected, simply connected, nilpotent Lie group $G$ and $\lambda$ be a unitary character of $H$. Assume that the irreducible unitary representation $\pi$ of $G$ is contained in $\operatorname{ind}(H, G, \lambda)$ and that $\pi \mid H$ contains $\lambda$. Then

i) the orbit $O_{\pi} \in \mathfrak{g}^{*} / \mathrm{Ad}^{*}(G)$ associated with $\pi$ is a linear variety in $\mathfrak{g}^{*}$;

ii) $H=G_{f}$ for any $f \in O_{\pi}$;

iii) $\operatorname{ind}(H, G, \lambda)$ is a multiple of $\pi$.

Proof. We begin by deriving some consequence of the above assumptions.

1) First we shall prove that $H$ is a normal subgroup of $G$ and that $\lambda_{g}=\lambda$ for all $g \in G$.

Applying 3.2 we get the direct integral descomposition

$\operatorname{ind}(H, G, \lambda) \mid H=\int_{H \backslash G / H}^{\oplus} \operatorname{ind}\left(H \cap H_{g}, H, \lambda_{g} \mid H \cap H_{g}\right) d v(\dot{g})$.

Now, the exponential map being a diffeomorphism for simply connected nilpotent Lie groups, $H \cap H_{g}=\operatorname{exph} \cap \exp \operatorname{Ad}(g)^{-1} \mathfrak{h}=\exp \left(\mathfrak{h} \cap \operatorname{Ad}(g)^{-1} \mathfrak{h}\right)$, where $\mathfrak{h}$ stands for the Lie algebra of $H$; in particular $H \cap H_{g}$ is connected. Since $\lambda$ is contained in ind $(H, G, \lambda) \mid H$, by 3.3 and 3.4, it results that $H_{g}=H$ and $\lambda_{g}=\lambda$ for all $g$ in a measurable subset of strictly positive Haar measure in $G$. It follows further that the closed subgroup of $G$ consisting of those $g \in G$ such that $H_{g}=H$ and $\lambda_{g}=\lambda$ is not negligeable with respect to the Haar measure of $G$. Consequently, it must be the whole $G$.

2) Let $\mathfrak{h}$ be the ideal of $\mathfrak{g}$ corresponding to $H$ and let $l \in \mathfrak{h}^{*}$ be the functional which corresponds to $\lambda$; that is $\lambda(\exp y)=e^{i l(y)}$ for all $y \in \mathfrak{h}$. We shall prove that $\mathfrak{f}$ $=\operatorname{Ker}(l)$ is an ideal of $\mathfrak{g}$.

Indeed, if $x \in \mathfrak{g}$ and $y \in \mathfrak{h}$, then $e^{i s l(\operatorname{Ad}(\exp t x) y)}=e^{i l(\operatorname{Ad}(\exp t x) s y)}=\lambda(\exp (t x) \exp (s y) \exp$ $(-t x))=\lambda(\exp s y)=e^{i s l(y)}$ for all $s \in \mathbb{R}$. Hence $l(\operatorname{Ad}(\exp t x) y)=l(y)$ for all $t \in \mathbb{R}$ and this implies that $(\operatorname{ad} x)^{m} y \in \mathfrak{f}$ for any integer $m \geqq 1$. In particular $[x, y] \in \mathfrak{f}$. $G_{f}$.

3) For all $f \in O_{\pi}, \mathfrak{h}$ is contained in the Lie algebra $\mathfrak{g}_{f}$ of the isotropy subgroup

To prove this assertion we note first that, since $\pi$ is contained in $\operatorname{ind}(H, G, \lambda)$ and $H$ is normal in $G$, we may apply Theorem 1 in [7] to deduce that there exists an $f_{0} \in O_{\pi}$ such that $f_{0} \mid \mathfrak{h}=l$. Now let $f=\operatorname{Ad}^{*}(g) f_{0}$ be arbitrary in $O_{\pi}$ and choose $x \in \mathfrak{g}$ such that $\exp x=g$. For any $y \in \mathfrak{h}$, in view of 2$)$, we have $f(y)=\sum_{m}(-1)^{m} / m$ ! $f_{0}\left((\operatorname{ad} x)^{m} y\right)=f_{0}(y)$. On the other hand, 2) also implies that $[\mathfrak{g}, \mathfrak{h}] \subset \mathfrak{f}$. Consequently, $f|[\mathfrak{g}, \mathfrak{h}]=l|[\mathfrak{g}, \mathfrak{h}] \subset l \mid \mathfrak{f}=0$. This means exactly that $\mathfrak{h} \subset \mathfrak{g}_{f}$.

4) The ideal $\mathfrak{h}$ contains the center $z$ of $g$ and the restriction of $\lambda$ to the center $Z$ of $G$ coincides with the central character $\lambda_{\pi}$ of $\pi$.

Indeed $\pi \mid Z$ which is a multiple of $\lambda_{\pi}$, is contained in $\operatorname{ind}(H, G, \lambda) \mid Z$ which by 3.2 is a multiple of ind $(Z \cap H, Z, \lambda \mid H \cap Z)$. The assertion follows now from 3.4.

5) We are now ready to finish the proof of the lemma, reasoning by induction on the dimension of $G$.

Let us note first that when $G$ is abelian $O_{\pi}$ reduces to a point so that i) trivially holds, while the remainder assertions ii) and iii) are also obviously true, by 3.4 for instance. Suppose now that $\mathfrak{f} \neq 0$ and let us denote $K=\exp (\mathfrak{f})$. Using 3 ), we see that $\lambda$, $\pi$, and ind $(H, G, \lambda)$ are trivial when restricted to $K$. Consequently, they can be viewed 
as being representations of the lower dimensional group $G / K$ for which the lemma is true by the induction hypothesis. It remains to handle the case when $\mathfrak{f}=0$. Then $\mathfrak{h} \neq 0$, otherwise the regular representation of $G$ would contain an irreducible unitary representation, which is definitely false. Therefore $\operatorname{dim}(\mathfrak{h})=1$. Then, according to 4$), \mathfrak{z}=\mathfrak{h}$ and $\operatorname{dim}(\mathfrak{z})=1$. The statement follows now immediately from [6].

\section{4.}

The results in the nilpotent case together with the Kirillov-Konstant principle (see [4]), which asserts that there should be a sort of correspondence between the unitary dual of a connected Lie group and the space of orbits of its coadjoint representation, suggest the following way to define intrinsically the concept of a "coherent state representation".

4.1. Definition. Let $G$ be a connected, simply connected Lie group. We shall say that the irreducible unitary representation $\pi$ of $G$ is a coherent state representation if it admits a system of coherent states based precisely on the "corresponding" orbit $O_{\pi} \in \mathfrak{g}^{*} / \operatorname{Ad}^{*}(\mathrm{G})$.

In the special case when $G$ is nilpotent, combining Brezin's Theorem 1.1 in [1] and the results in Sections 2 and 3 above, we get the following characterization of coherent state representations.

4.2. Theorem. An irreducible unitary representation $\pi$ of a connected and simply connected nilpotent Lie group $G$ is a coherent state representation if and only if it is a square integrable representation of the quotient group $G / \operatorname{Ker} \pi$.

One may plausible conjecture that this assertion retains its validity for exponential solvable Lie groups. Finally we note that it is patently true for compact groups.

Acknowledgements. I want to thank my colleague Horia Scutaru for many constructive discussions on coherent state representations.

\section{References}

1. Brezin, J. : Geometry and the method of Kirillov. In : Lecture notes in mathematics, No. 466, p. $13-26$. Berlin-Heidelberg-New York: Springer 1975

2. Dixmier,J.: Représentations induites holomorphes des groupes résolubles algébriques. Bull. Soc. Math. France 94, 181-206 (1966)

3. Kirillov, A. A. : Unitarnie predstavlenia nilpotentih grupp Li. Uspehi Mat. Nauk 17, 57-110 (1962)

4. Kostant,B.: Quantization and unitary representations. I. Prequantization. In: Lecture notes in mathematics, No. 170, pp. 87-208. Berlin-Heidelberg-New York: Springer 1970

5. Mackey, G. W. : Induced representations of locally compact groups. I. Ann. Math. 55, 101-130 (1952)

6. Moore,C.C., Wolf,J.A.: Square integrable representations of nilpotent groups. Trans. Amer. Math. Soc. 185, 445-462 (1973)

7. Moscovici,H.: Topological Frobenius properties for nilpotent Lie groups. Rev. Roum. Math. Pures Appl. 19, 421-425 (1974)

8. Perelomov, A. M.: Coherent states for arbitrary Lie groups. Commun. math. Phys. 26, 222-236 (1972)

Communicated by H. Araki

Received January 30, 1976; in revised form November 9, 1976 